\title{
Measuring temperature in the lens during experimental heat load indirectly as light scattering increase rate
}

Zhaohua Yu

Nooshin Talebizadeh

Martin Kronschläger

Per Söderberg 


\title{
Measuring temperature in the lens during experimental heat load indirectly as light scattering increase rate
}

\author{
Zhaohua Yu, ${ }^{*}$ Nooshin Talebizadeh, Martin Kronschläger, and Per Söderberg \\ Uppsala University, Department of Neuroscience, Gullstrand Lab. Ophthalmology, Uppsala 751 85, Sweden
}

\begin{abstract}
The current study aims to experimentally estimate the temperature in the lens due to heat load indirectly from the measurement of increases in the rate of temperature-induced light scattering. The lens was extracted from Sprague-Dawley rats and put into a temperature-controlled cuvette filled with a balanced salt solution. Altogether, 80 lenses were equally divided into four temperature groups. Each lens was exposed for $5 \mathrm{~min}$ to temperature depending on the group to which it belonged while the intensity of forward light scattering was recorded. The inclination coefficients of light scattering increase at the temperature of $37^{\circ} \mathrm{C}, 40^{\circ} \mathrm{C}, 43^{\circ} \mathrm{C}$, and $46^{\circ} \mathrm{C}$ were estimated as a $\mathrm{Cl}(0.95), 3.1 \pm 0.8,4.4 \pm 0.8,5.5 \pm 0.9$, and $7.0 \pm 0.8 \times 10^{-4} \mathrm{tEDC} / \mathrm{s}$, respectively. The Arrhenius equation implies that the natural logarithm of the inclination coefficient is linearly dependent on the inverse of the temperature. The proportionality constant and the intercept were $9.6 \pm 2.4 \times 10^{3} \mathrm{~K}$ and $22.8 \pm 7.7$, respectively. The activation energy was $8.0 \pm 2.0 \times 10^{1} \mathrm{~kJ} \cdot \mathrm{mol}^{-1}$. The current experiment implies that if averaging 20 measurements of inclination coefficients in a new experiment at constant heat load, the confidence limits for predicted temperature correspond to $\pm 1.9^{\circ} \mathrm{C}$. With the proportionality constant and the intercept estimated in the current experiment, the in vivo temperature in the lens can be determined retrospectively with sufficient resolution. ๑ 2017 Society of Photo-Optical Instrumentation Engineers (SPIE) [DOI: 10.1117/1.JBO.22 .1.015005]
\end{abstract}

Keywords: forward light scattering; lens; cataract; temperature; Arrhenius equation.

Paper 160459RR received Jul. 1, 2016; accepted for publication Jan. 10, 2017; published online Jan. 31, 2017.

\section{Introduction}

Thermal cataracts were first identified in the late 1800s. ${ }^{1}$ Both clinical observations of cataracts in glassblowers and steel workers and experimental studies have indicated an association between thermal exposure and cataract formation. ${ }^{2-4}$

To measure the in vivo temperature in the lens during thermal exposure is challenging. A thermocouple probe damages the lens and decreases the heat load due to heat conduction in the probe. ${ }^{5-7}$ Fiber optic sensors are based on fluorescence excited in the near-infrared waveband and are therefore unsuitable for temperature measurement due to infrared radiation heat load.

Clinically, a cataract infers permanent light scattering in the lens that decreases vision perception. To predict permanent light scattering in the lens induced by thermal exposure, experimentally or by modeling, it is important to know the critical temperature for permanent light scattering in the lens. The critical temperature for permanent light scattering in the lens is currently unknown because of the difficulties in measuring the in vivo temperature during thermal exposure.

The Arrhenius equation models the denaturation rate as a function of temperature. On the assumption that light scattering in the lens depends on denaturation of lens proteins, the Arrhenius equation can be applied to experimentally establish the relationship between temperature at exposure and rate of

*Address all correspondence to: Zhaohua Yu, E-mail: Zhaohua.yu@ neuro.uu se light scattering increase (Appendix A). The long-term goal is to establish data on in vivo lens protein denaturation after heat load that allows rational criteria for safe exposure to infrared radiation.

The purpose of this study was to explore the feasibility for experimental indirect estimation of lens temperature based on light scattering measurement in the lens.

\section{Materials and Methods}

\subsection{Animals}

Six-week-old albino Sprague-Dawley female rats were kept and treated according to the ARVO Statement for the Use of Animals in Ophthalmic and Vision Research. Ethical approval was obtained by Uppsala Djurförsöksetiska Nämnd (C29/16).

\subsection{Temperature Measurement}

Temperature was measured with manufacturer calibrated thermocouples (HYP0, OMEGA) connected to an integrated analogue-digital converter (TC-08, OMEGA).

\subsection{Measurements of Forward Light Scattering in the Lens}

The intensity of forward light scattering in the lens was measured on a dark field source (Fig. 1). After reflection with

1083-3668/2016/\$25.00 @ 2016 SPIE 


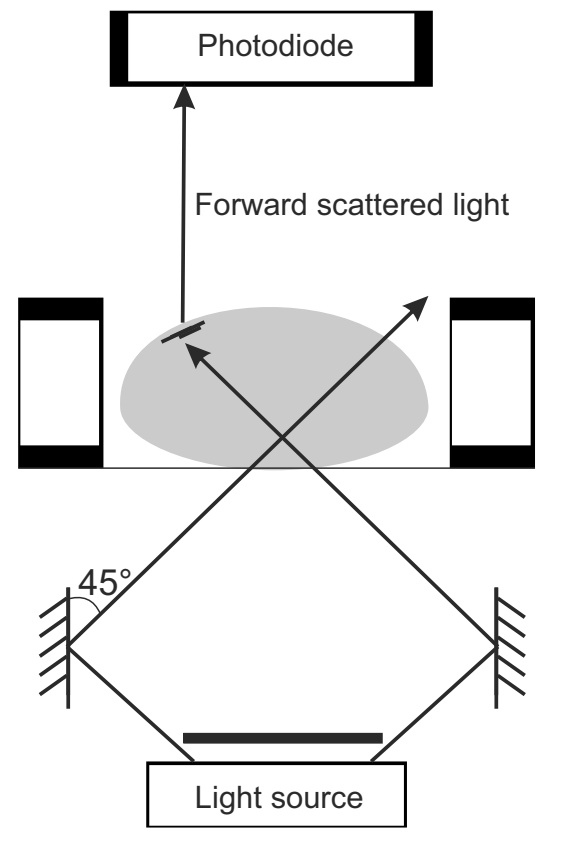

Fig. 1 Schematic of device for quantitative measurement of the intensity of forward light scattering in the lens.

mirrors, the light from the dark field source hits the cuvette cavity in a narrow circular beam from below at a $45-\mathrm{deg}$ angle. The cuvette is imaged on a photodiode, which generates a current. Current readings are converted according to a calibration curve to the concentration of a known light scattering standard concentration, a commercial lipid emulsion of Diazepam (Stesolid Novum, Actavis AB, Sweden). To make measurements normally distributed, the concentrations of Diazepam are $\log$ transformed, as transformed equivalent diazepam concentration (tEDC). ${ }^{8}$

\subsection{Exposure Setup}

The exposure setup consisted of a cuvette that has an inner water channel bypassing a central well (Fig. 2). A pump (MD-10, IWAKI Co., Japan) drives water from a temperature-controlled water bath (VWB 12, VWR, Germany) in a closed loop through a heater, indirectly regulating the temperature in the lens cuvette filled with a sterile balanced salt solution (BSS: BSS Sterile Irrigating Solution, Alcon). (a)

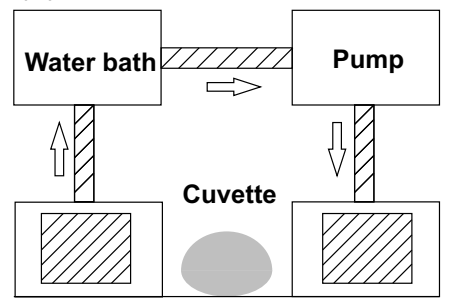

(b)

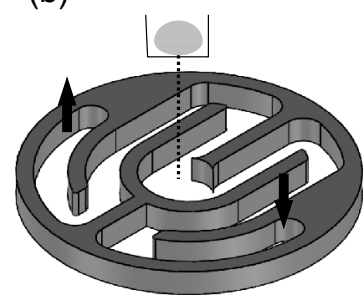

Fig. 2 Schematic of temperature-controlled lens cuvette. (a) Water bath and associated pump driving temperature-controlled water through a heater, indirectly regulating the temperature in the lens cuvette filled with BSS and (b) water channel bypassing the cuvette. Arrows indicate in and out flow of circulating water.

\subsection{Experimental Procedure}

The temperature-controlled cuvette filled with BSS was preheated by the circulating water to the planned exposure temperature. The temperature was confirmed by the thermocouple.

The animal was sacrificed. Then one lens was extracted and put into the preheated BSS in the cuvette; intensity of forward light scattering in the lens was measured for $5 \mathrm{~min}$, as described elsewhere. This assured a fast increase of lens temperature.

\subsection{Experimental Design}

Altogether, 80 lenses from 80 animals were equally divided into four temperature groups, $37^{\circ} \mathrm{C}, 40^{\circ} \mathrm{C}, 43^{\circ} \mathrm{C}$, and $46^{\circ} \mathrm{C}$.

\subsection{Statistical Parameters}

The significance limit and the confidence level were set to 0.05 and 0.95 , respectively, considering the sample size.

\section{Results}

\subsection{Light Scattering Increase as a Function of Exposure Time}

The intensity of light scattering, $\Delta I_{s}$, recorded at exposure times between 30 and $300 \mathrm{~s}$ was fit to a linear model [Eq. (1)], considering an initial light scattering, $k_{0}$ (tEDC), and an inclination coefficient, $k_{1}(\mathrm{tEDC} / \mathrm{s})$

$\Delta I_{s}=k_{0}+k_{1} \cdot t$.

The average light scattering increased temperature dependently as a function of time (Fig. 3).

The averaged inclination coefficient ranged between 3.1 and $7.0 \times 10^{-4} \mathrm{tEDC} / \mathrm{s}$ depending on incubation temperature (Table 1).

\subsection{Natural Logarithm of the Inclination Coefficient for Light Scattering Increase as a Function of the Inverse of the Absolute Temperature}

The natural logarithm of the inclination coefficient for light scattering increase, as a function of the inverse of the absolute temperature, was fit to a linear model [Appendix Eq. (9), Fig. 4].

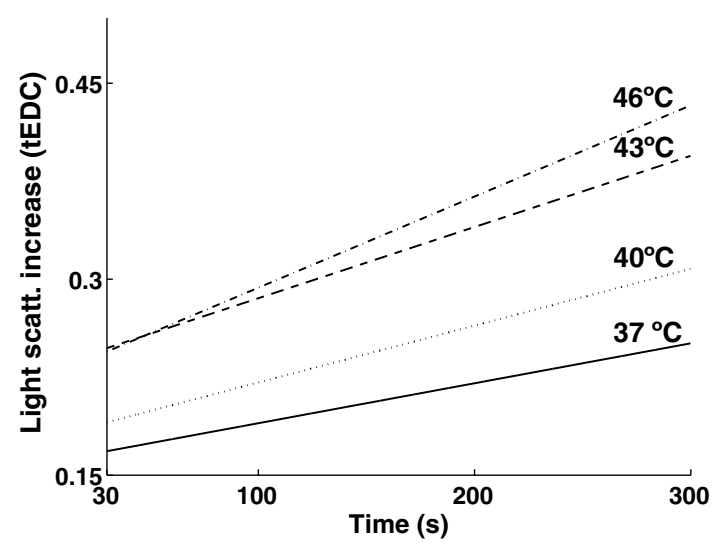

Fig. 3 Averaged intensity of light scattering as a function of exposure time from 30 to $300 \mathrm{~s}$ in lenses exposed to the temperatures in the interval 37 to $46^{\circ} \mathrm{C}$. 
Table 1 Estimated inclination coefficients for evolution of light scattering in the lens as a function of time and their dependence on temperature.

\begin{tabular}{lc}
\hline & \multicolumn{1}{l}{ Inclination coefficient $\times 10^{-4}(\mathrm{tEDC} / \mathrm{s})$} \\
\cline { 2 - 2 } Temperature $\left({ }^{\circ} \mathrm{C}\right)$ & $95 \%$ confidence interval for the mean \\
\hline 37 & $3.1 \pm 0.8$ \\
40 & $4.4 \pm 0.8$ \\
43 & $5.5 \pm 0.9$ \\
46 & $7.0 \pm 0.8$ \\
\hline
\end{tabular}

${ }^{a}$ d.f. $=19$.

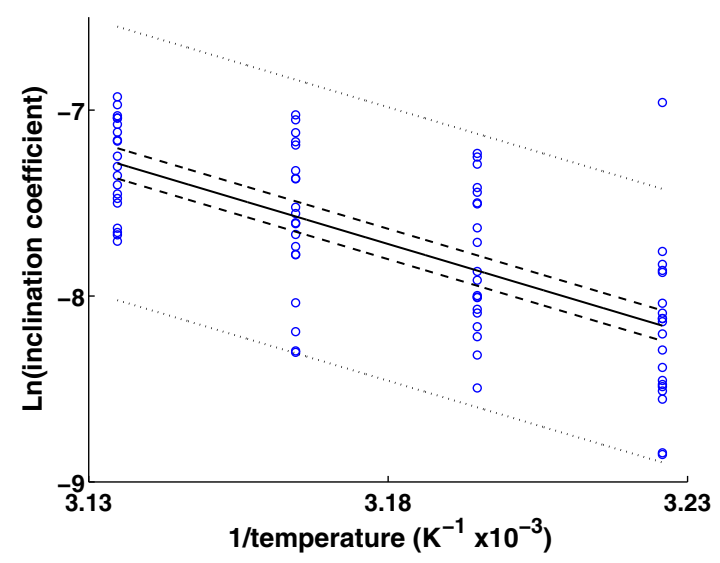

Fig. 4 The natural logarithm of the inclination coefficient for light scattering increase as a function of the inverse of the absolute temperature. Open circles are estimates of the natural logarithm of the inclination coefficient for each lens. Continuous line is the regression line. Dashed lines are $\mathrm{Cl}(0.95)$ for the regression line. Dotted lines are $\mathrm{Cl}(0.95)$ for one additional measurement of inclination coefficient for prediction of the inverse temperature.

The proportionality constant $E_{a} / R\left(E_{a}=\right.$ the activation energy, $R=$ the general gas constant) and the intercept $\ln (l \cdot P)$ were estimated as $\mathrm{CI}(0.95), \quad 9.6 \pm 2.4 \times 10^{3} \mathrm{~K}$ and $22.8 \pm 7.7$. Since $R$ is known, $E_{a}$ was estimated as a $\mathrm{CI}(0.95), 8.0 \pm 2.0 \times$ $10^{1} \mathrm{~kJ} \mathrm{~mol}^{-1}$.

A calibration curve for dependence of rate of light scattering increase (here, inclination coefficient) on temperature can be established experimentally. The confidence interval for predicted temperature can be estimated from an average of inclination coefficients. ${ }^{9}$ The precision depends on the residual variance, the sample size constituting the basis of the average, and the number of additional measurements of inclination coefficients averaged. The lowest precision is associated with one additional measurement of inclination coefficient in a future experiment for temperature probing (Fig. 4).

\subsection{Simulation of Sample Size Required to Estimate Temperature Based on Inclination Coefficient}

To judge the practical validity of the described strategy, it is necessary to estimate the precision of predicted temperature as a function of sample size. Adopting the outcome of the current

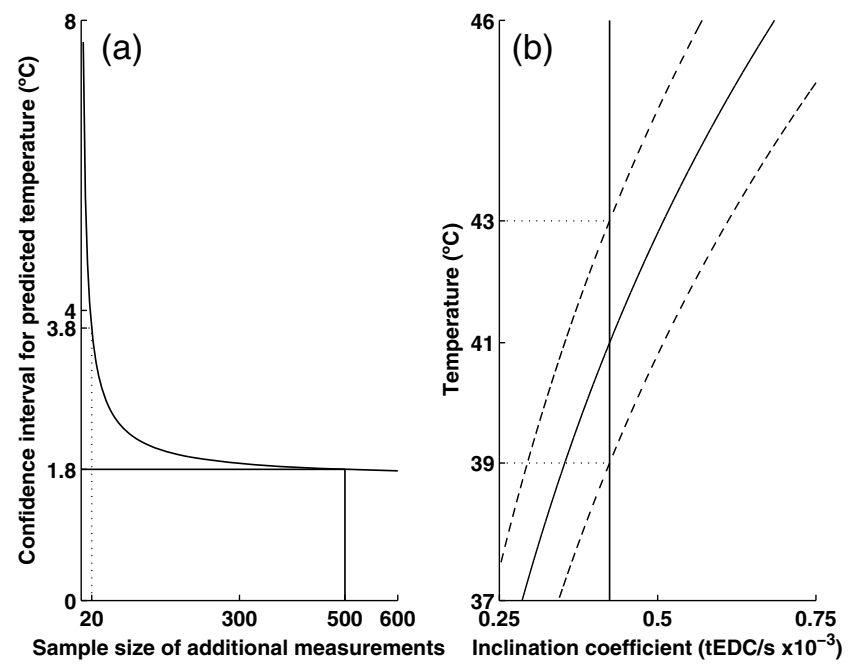

Fig. 5 (a) The estimated confidence interval for predicted temperature as a function of sample size of additional measurements, adopting the outcome of the estimates of inclination coefficients for increase of light scattering as a function of time and temperature. (b) Continuous line is the regression line for predicted temperature as a function of inclination coefficient. Dashed lines are $\mathrm{Cl}(0.95)$ for the regression line. Vertical line corresponds to an example of an estimated inclination coefficient averaged from 20 additional measurements. Horizontal lines indicate the associated confidence limits for predicted temperature.

estimates of inclination coefficients for an increase of light scattering as a function of time and temperature, the confidence interval for predicted temperature based on averages of additional measurements of inclination coefficients can be estimated. ${ }^{9}$ It was found that averaging 20 additional measurements of inclination coefficients, the confidence limits around the estimated temperature correspond to $\pm 1.9^{\circ} \mathrm{C}$ (Fig. 5).

Averaging a very large sample of additional measurements, the estimated confidence limits for the estimated temperature approximate $\pm 0.9^{\circ} \mathrm{C}$.

\section{Discussion}

The current study intended to explore the possibility of using denaturation as an indirect method to experimentally measure lens temperature due to heat load, applying the Arrhenius equation. Denaturation was measured as light scattering.

To allow temperature measurement based on denaturation, the time constant for heat transfer must be small in relation to the time constant for denaturation. An estimate assuming Newton's law of heating indicated that for the current experiment this requirement holds.

The light scattering measurement was recorded for $5 \mathrm{~min}$ during exposure. At the beginning of exposure, the heat transfer to the lens still occurred. Therefore, the intensity of forward light scattering in the time window 30 to 300 s was selected for fitting light scattering as a function of time with regression.

To allow for a short time constant for temperature equilibrium, a small mass should be heated. Therefore, the rat lens was selected for this experiment.

An in vitro study of cold cataracts indicated that temperatureinduced light scattering varies little in the range from 16 to $37^{\circ}$ C. ${ }^{10}$ In a pre-experiment, we found that the inclination coefficient for light scattering increases as a function of time up to a temperature of $48^{\circ} \mathrm{C}$ and approximates an asymptote toward 
higher temperatures. A study of lenses from multiple species showed that at temperatures between $55^{\circ} \mathrm{C}$ and $65^{\circ} \mathrm{C}$ most of the soluble proteins are lost. ${ }^{11}$ In the present investigation, we aimed at temperatures just above the threshold for denaturation. Therefore, the minimum exposure temperature was set to $37^{\circ} \mathrm{C}$ and the maximum was set to $46^{\circ} \mathrm{C}$.

The finding that the inclination coefficient for light scattering increase increased with temperature (Fig. 3 and Table 1) agrees with the Arrhenius equation (Appendix A). Therefore, for the selected time and temperature range, it is possible to estimate temperature in the lens with light scattering increase rate.

Considering the sample size in the current experiment, the confidence interval for the inclination coefficient as a function of temperature (Table 1) reflects a substantial variation of scattering-time response among lenses from different animals. The temperature in the cuvette was controlled to within $\pm 0.1^{\circ} \mathrm{C}$. Therefore, the observed variation in scattering-time response most probably reflects variability in temperature sensitivity in the lens among individuals. This is also revealed by the individual estimates of the natural logarithm of the inclination coefficient, plotted in Fig. 4. This may implicate a substantial variability in sensitivity to sudden heat load in the lens among individuals, which has to be considered when setting safety guidelines for human exposure. Considering the Arrhenius equation, denaturation rate is directly dependent on temperature. Therefore, it is possible that a very small heat load over a long time may accelerate cataract formation. ${ }^{12}$

The activation energy calculated for temperature-induced aggregation of protein in whole lens, $8.0 \pm 2.0 \times 10^{1} \mathrm{~kJ} \cdot \mathrm{mol}^{-1}$, based on the outcome depicted in Fig. 4, is in the range of what has previously been reported for temperature-induced conformational change of $\gamma$-crystalline tryptophan, ${ }^{13}$ temperatureinduced $\alpha$-crystalline aggregation, ${ }^{14}$ and chemically induced aggregation of $\gamma \mathrm{F}$-crystallin. ${ }^{15}$

The absolute resolution limit of the currently presented method is around $\pm 1{ }^{\circ} \mathrm{C}$ even if a very large sample size is averaged [Fig. 5(a)]. The resolution limit is determined by the variability in scattering-time response as a function of temperature among individuals (Fig. 4). However, with 20 additional measurements of inclination coefficients, the resolution is on the order of $\pm 2^{\circ} \mathrm{C}$. For experiments aiming at estimating lens sensitivity to heat load, $\pm 2^{\circ} \mathrm{C}$ is sufficient.

To determine the dependence of denaturation rate on heat load in vivo, it would be necessary to measure denaturation rate as heat-induced rate of back scattering. In vitro determined dependence of denaturation rate on temperature can be used to estimate the heat load-induced lens temperature. Consequently, the relationship between temperature in the lens and in vivo heat load exposure of the eye can be determined. Then, the critical temperature in the lens can be estimated by in vivo exposures at incrementing heat load exposure of the eye with subsequent postexposure measurements of permanent light scattering in the lens (Fig. 6).

Such measurements of permanent light scattering should be done at postexposure time intervals long enough to exclude immediate reversible light scattering. Knowledge about experimentally determined critical temperature in lenses from warmblooded animals can be used for extrapolation to human lens.

The currently presented method for measurement of lens temperature during heat load is to our knowledge the only available method that allows temperature measurement without disturbance from the measurement sensor.

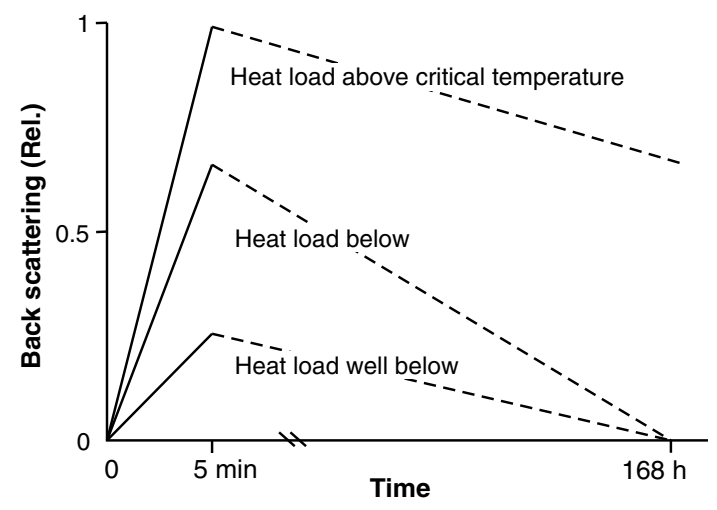

Fig. 6 Experimental model for estimation of critical temperature with incremental heat load.

\section{Appendix: Derivation of the Relationship Between Temperature and Light Scattering Measurement in the Lens}

\section{A1 Light Scattering as a Measure of Denaturation}

The relative intensity of scattered light, $I_{s}$, can be considered directly proportional to the concentration of denatured stated proteins, $\Omega\left(\mathrm{mol} \cdot \mathrm{l}^{-1}\right)$, with a proportionality constant, $l$ $\left(\mathrm{mol}^{-1} \cdot 1\right)$

$I_{s}=l \cdot \Omega$.

Experimentally, the difference of light scattering between temperature-induced intensity of light scattering and base line intensity of light scattering, $\Delta I_{s}$, is a measure of the temperature-induced concentration of denatured state proteins

$\Delta I_{s}=l \cdot \Omega$

or

$\Omega=\frac{\Delta I_{s}}{l}$.

\section{A2 Arrhenius Equation}

The rate of production of denatured proteins, $r\left(\mathrm{~mol} \cdot \mathrm{1}^{-1} \cdot \mathrm{s}^{-1}\right)$, is proportional to the concentration of nature state protein, $[N]$ $\left(\mathrm{mol} \cdot \mathrm{l}^{-1}\right)$, with a proportionality constant, $k(T)\left(\mathrm{s}^{-1}\right)$, that varies with temperature, $T(\mathrm{~K})$

$r=k(T)[N]$

or

$\frac{\mathrm{d} \Omega}{\mathrm{d} t}=k(T)[N]$

Arrhenius demonstrated that the reaction rate constant, $k(T)\left(\mathrm{s}^{-1}\right)$, varies exponentially with the inverse of the molecular enthalpy, the general gas constant, $R \cdot\left(8.31 \mathrm{~J} \cdot \mathrm{mol}^{-1} \cdot \mathrm{K}^{-1}\right)$, multiplied with the temperature, $T\left(\mathrm{~K}^{-1}\right)$, and a proportionality constant, the activation energy, $E_{a}\left(\mathrm{~J} \cdot \mathrm{mol}^{-1}\right)$, determined by a proportionality constant, $A\left(\mathrm{~s}^{-1}\right)$, the pre-exponential factor 
$k(T)=A e^{-\frac{E_{a}}{R T}}$

The pre-exponential factor expresses the maximum rate at infinitely high temperature and reflects the increase in entropy associated with the loss of order due to the reaction.

Inserting Eq. (5) into Eq. (4) results in Eq. (6):

$$
\frac{\mathrm{d} \Omega}{\mathrm{d} t}=A \cdot[N] \cdot e^{-\frac{E_{a}}{R T}}
$$

For a certain tissue, the original concentration of native state molecules is constant. Then, Eq. (7) can be simplified by substituting $A$ and $[N]$ with a rate constant, $P\left(\mathrm{~mol} \cdot \mathrm{1}^{-1} \cdot \mathrm{s}^{-1}\right)$

$$
\frac{\mathrm{d} \Omega}{\mathrm{d} t}=P \cdot e^{-\frac{E_{a}}{R T}}
$$

or

$$
\ln \frac{\mathrm{d} \Omega}{\mathrm{d} t}=\ln P-\frac{E_{a}}{R} \cdot \frac{1}{T} .
$$

At temperature saturation, heat application is in balance with heat loss through heat diffusion and convection. Thus, temperature at saturation, $T$, is independent of the exposure time, and the denaturation rate, $d \Omega / d t$, is directly proportional to the exposure time, $t$. Therefore, Eq. (7) can be rewritten as follows:

$$
\Omega=t \cdot P \cdot e^{-\frac{E_{a}}{R T}}
$$

or

$$
\ln (\Omega)=\ln (t)+\ln (P)-\frac{E_{a}}{R} \cdot \frac{1}{T} .
$$

Or after substitution of $\Omega$ according to Eq. (3) as Eq. (9):

$$
\frac{\Delta I_{s}}{l}=t \cdot P \cdot e^{-\frac{E_{a}}{R T}}
$$

or

$$
\frac{\Delta I_{s}}{t}=(l \cdot P) \cdot e^{-\frac{E_{a}}{R T}}
$$

or

$\ln \left(\frac{\Delta I_{s}}{t}\right)=\ln (l \cdot P)-\frac{E_{a}}{R} \cdot \frac{1}{T}$.

Thus, $\ln \left(\Delta I_{s} / t\right)$ is linearly dependent on $1 / T$ with the proportionality constant $E_{a} / R$ and the intercept $\ln (l \cdot P)$.

\section{Disclosures}

The authors have no conflicts of interest, financial or otherwise, and no competing interests.

\section{Acknowledgments}

The current study was supported by Carmen och Bertil Regnérs fond för forskning, Gun och Bertil Stohnes Stiftelse, Karin
Sandqvists Stiftelse, Svenska Läkaresällskapet Resebidrag, Konung Gustav V:s och Drottning Victorias Frimurarstiftelse, Uppsala Läns Landsting's Research grants (ALF), Ögonfonden, Stiftelsen Sigurd och Elsa Goljes Minne.

\section{References}

1. W. Meyenhofer, "Zur Aetiolgie des graven staars: Jugendliche kataraken bei glasmachern," Klin Monatabl Augenheilk 24, 49-67 (1886).

2. F. H. Verhoeff, L. Bell, and C. B. Walker, "The pathological effects of radiant energy upon the eye," Proc. Am. Acad. Arts Sci. 51, 629-818 (1915).

3. J. Wallace, "An epidemiological study of lens opacities among steelworkers," Br. J. Ind. Med. 28, 265-271 (1971).

4. E. Lydahl, "Ocular exposure to infrared radiation in the Swedish iron and steel industry," Health Phys. 46, 529-536 (1984).

5. Z. Yu et al., " $1090 \mathrm{~nm}$ infrared radiation at close to threshold dose induces cataract with a time delay," Acta Ophthalmol. 93(2), e118-e122 (2015).

6. Z. Yu et al., "Ocular temperature elevation induced by threshold in vivo exposure to 1090-nm infrared radiation and associated heat diffusion," J. Biomed. Opt. 19(10), 105008 (2014).

7. Z. Yu et al., "Temperature-controlled in vivo ocular exposure to 1090$\mathrm{nm}$ radiation suggests that near-infrared radiation cataract is thermally induced," J. Biomed. Opt. 20(1), 015003 (2015).

8. P. G. Söderberg, E. Chen, and B. Lindström, "An objective and rapid method for the determination of light dissemination in the lens," Acta Ophthalmol. Copenh 68, 44-52 (1990).

9. J. H. Zar, "Ch. 17.4 confidence intervals in regression," in Biostatistical Analysis, pp. 337-342, Prentice Hall, Upper Saddle River (1999).

10. V. Petta et al., "Dynamic light scattering study on phase separation of a protein-water mixture: application on cold cataract development in the ocular lens," Phys. Rev. E 77(6 Pt 1), 061904 (2008).

11. M. J. McFall-Ngai and J. Horwitz, "A comparative study of the thermal stability of the vertebrate eye lens: Antarctic ice fish to the desert iguana," Exp. Eye Res. 50(6), 703-709 (1990).

12. D. H. Sliney, "Physical factors in cataractogenesis: ambient ultraviolet radiation and temperature," Invest. Ophthalmol. Vis. Sci. 27, 781-790 (1986).

13. R. F. Borkman, A. Douhal, and K. Yoshihara, "Picosecond fluorescence decay of lens protein $\gamma$-II crystallin," Biophys. Chem. 47(3), 203-211 (1993).

14. G. Maulucci et al., "The thermal structural transition of $\alpha$-crystallin inhibits the heat induced self-aggregation," PLoS One 6(5), e18906 (2011).

15. B. K. Das and J. J.-N. Liang, "Thermodynamic and kinetic characterization of calf lens $\gamma$ F-crystallin," Int. J. Biol. Macromol. 23(3), 191-197 (1998).

Zhaohua Yu, MD, PhD, concentrates on the damage mechanism of near-IRR cataracts.

Nooshin Talebizadeh, MD, PhD, majors in caspase-3 in ultraviolet radiation cataracts.

Martin Kronschläger, MD, PhD, studies prevention of experimental cataracts induced by ultraviolet radiation.

Per Söderberg is a professor and chair of ophthalmology at Uppsala University. He focuses on the interaction of optical radiation and the eye, cataract measurement, contrast sensitivity, virtual reality cataract surgery, corneal endothelium biometry, and digital visual acuity charts. 